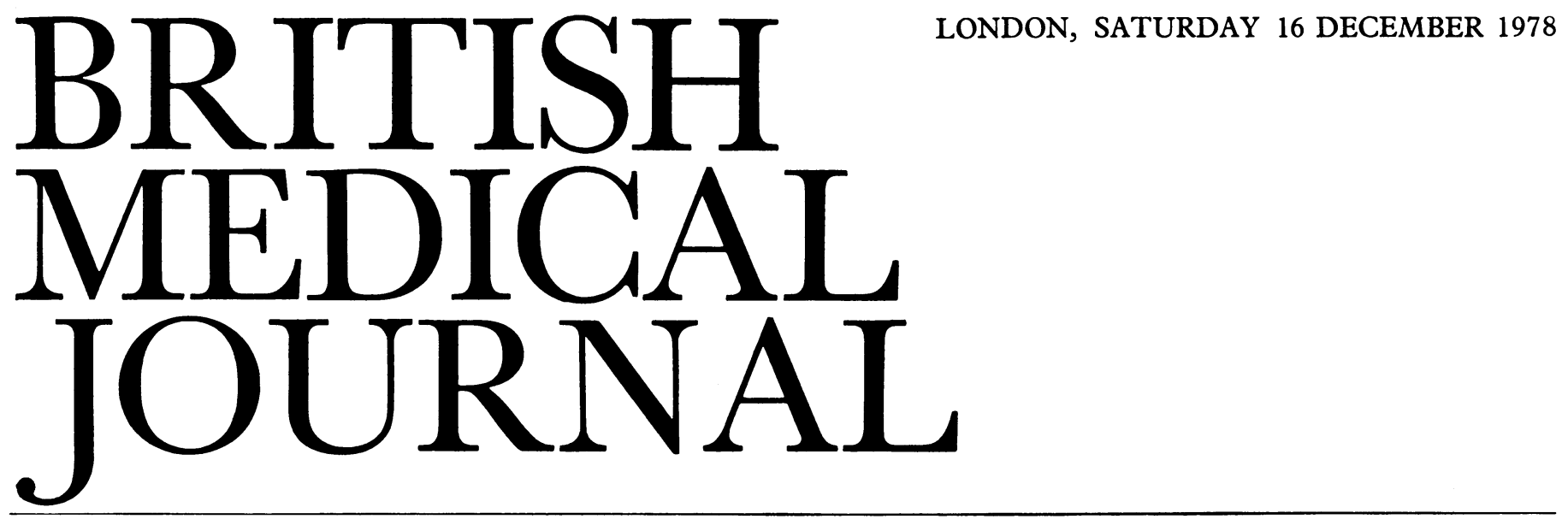

\title{
Hazards of amniocentesis
}

The antenatal diagnosis of fetal defects is perhaps the greatest advance in perinatal medicine for a generation. Enthusiasts speak $^{1}$ of the eventual possibility of removing serious congenital anomalies from the childhood population: "The birth of children suffering from chromosome anomalies or spina bifida could be prevented by carrying out amniocentesis on all pregnant women." Given that all pregnant women seek antenatal care before 16 weeks, that Utopian dream requires two main ingredients: a safe and effective diagnostic procedure available to the whole childbearing population, and the legal sanction to terminate pregnancy when the diagnosis has been made.

Given the enormous resources in cash and manpower that would be needed for amniocentesis to be made available to all pregnant women, can it be said to be safe ? Case reports of hazards associated with amniocentesis in mid-pregnancy have been balanced by reassuring (if statistically somewhat unsatisfactory) surveys from Europe ${ }^{2}$ and America $^{3}$ and so led to uncertainty; but the publication ${ }^{4}$ of a report by the Medical Research Council Working Party on Amniocentesis should remove many of the doubts. This rigorously controlled study in nine British obstetric centres was based on 2428 women who had a diagnostic amniocentesis before 20 weeks of pregnancy between January 1973 and September 1976, and 2428 matched controls; the children born were followed up until the age of 10 months. The study took place before ultrasound had become universally available, so that only a minority of amniocenteses were performed under ultrasound control, and in a substantial number of cases there was not even an attempt to locate the placenta; that was a pity, but it does not invalidate the main conclusions.

The report must be read in all its detail and complexity by every practising obstetrician, but in essence the hazards that were clearly apparent were an excess risk of abortion, estimated to be between $1 \%$ and $1.5 \%$; an excess risk of serious antepartum haemorrhage of about $1 \%$, with the increase occurring in both abruption and placenta praevia; an excess risk of about $1 \%$ of the infant's having unexplained respiratory difficulties at birth lasting for more than 24 hours; a similar excess of major orthopaedic postural deformities, largely severe talipes equinovarus and congenital dislocation of the hip; and an increased risk of rhesus isoimmunisation. Overall perinatal mortality was 20 in the amniocentesis group and 11 in the controls.

Apart from the increased risk of abortion and of rhesus sensitisation these hazards were unexpected, but the evidence indicates that they were not chance phenomena and they must be explained. For the increase in antepartum haemorrhage, and particularly of placenta praevia, no simple explanation is apparent; but the possibility that damage to the uterine wall could play a part deserves investigation. The increase in respiratory difficulties and of orthopaedic defects strongly suggests the effects of uterine pressure on a fetus unprotected by its usual cushion of fluid. In theory the removal of less than $20 \mathrm{ml}$ of amniotic fluid from an average of about $200 \mathrm{ml}$ at 16 weeks seems unlikely to be harmful, but fluid may leak away from the site of puncture, and there could be a serious loss in the hours and days after amniocentesis. Modern ultrasound techniques should be capable of measuring that loss.

The working party concluded that in each individual patient the assessment of the value and acceptability of amniocentesis "must depend on a comparison between the chance of detecting a fetal abnormality and the hazards of the procedure. Our results show that the balance of advantage depends critically upon the indication for amniocentesis. Thus, when it was undertaken because a previous sibling had been affected by a single autosomal gene defect, or because the mother was a translocation carrier for Down's syndrome, or for a raised level of maternal serum alpha fetoprotein, the chance of detecting a serious abnormality was substantially greater than the estimated increase in fatal and non-fatal effects on the fetus. But for other indications, the balance of advantage was less clear. In particular, it was only at 40 years or more that maternal age alone stood out clearly as an indication in which the chances of detecting an abnormality definitely outweighed the increase in risks to the fetus." A similar type of accounting, based not on hazards but on financial costs, was made by Hagard and Carter ${ }^{5}$ they calculated that the potential economic benefits of detecting Down's syndrome and terminating the pregnancy were considerable in women of 40 or more but diminished at lower ages, so that in women under 35 the costs of screening would exceed the economies achieved in health care and education.

Such conclusions assume that the terms in the equation are equivalent-and they are not. The cost of serious spina bifida is much more than any financial expense: it is the cost of the anguish and disruption of an entire family and the cost of a cheerless existence for the child itself. Who is to say that those "costs" are more or less than the "cost" of losing a pregnancy by abortion or stillbirth, or the "cost" of a correctable dislocation of the hips? Only the individual 
parents can make that decision, and with the information from this report their obstetrician is now in a better position to guide them.

${ }^{1}$ British Medical fournal, 1977, 1, 1430.

2 Galjaard, H, Cytogenetics and Cell Genetics, 1976, 16, 453.

${ }^{3}$ United States National Institute of Child Health and Human Development Study Group, fournal of the American Medical Association, 1976, 236, 1471.

${ }^{4}$ Medical Research Council Working Party on Amniocentesis, British fournal of Obstetrics and Gynaecology, 1978, 85, suppl 2 .

${ }^{5}$ Hagard, S, and Carter, F A, British Medical fournal, 1976, 1, 753.

\section{Viruses and reclaimed water}

The quantity of water a person uses each day varies from five litres for the more primitive nomads to 500 litres in economically developed countries. Yet only $20^{\circ}$ of the world's population has a direct supply-and for the population of the developed nations the figure is only $5 \% .{ }^{1}$ The demands on water are increasing so rapidly because of population expansion and industrial needs that recycling of domestic waste and sewage is practically inevitable in the future. The presence of viruses in sewage effluent, streams and rivers, lakes, and other waters is an important health hazard with the increasing contamination of surface water.

Over 100 viruses are excreted in human faeces, including poliovirus, echoviruses, coxsackieviruses, hepatitis A virus, viruses causing gastroenteritis, and certain adenovirus serotypes. In fact, well over one million virus particles may be excreted per gram of faeces, and concentrations as high as 500000 infectious virus particles per litre have been detected in raw sewage. ${ }^{2}$ Furthermore, sufficient numbers of enteric virus particles survive the customary secondary treatment of sewage and chlorination to be easily detected. Some of these viruses can survive for 25-125 days in soil, 2-168 days in tap water, and 2-130 days in sea water. Indeed, human viruses adsorbed to soil are infectious for animals and in tissue culture. Methods for removing or inactivating viruses that are adsorbed on to and within solids are therefore also important.

Inactivating viruses is clearly preferable to simply removing them from water by processes such as sedimentation, adsorption, coagulation and precipitation, and filtration. The mainstay of water disinfection has been chlorine treatment, but its effectiveness depends on many factors including temperature, $\mathrm{pH}$, and the presence of organic matter. High doses of chlorine are a hazard to various forms of life when the effluents are discharged: carcinogenic chlorinated hydrocarbons may be produced, and possibly (to judge by recent laboratory findings) a progressively more chlorine-resistant poliovirus strain could develop.

We know less about viruses in drinking water. Enteric viruses have been detected, however, in $18 \%$ of 200 samples in Paris; virus has been isolated from drinking water in the USSR, South Africa, and Romania ${ }^{2}$; and poliovirus has been detected on several occasions in treated drinking water containing free residual chlorine in the United States. ${ }^{3}$ But viruses may be transmitted to man not only by contaminated drinking water but also by shellfish and crops; and swimming and other water recreations may provide further sources of infection. Moreover, spraying crops with waste water in agricultural settlements in Israel has recently been found to produce aerosols contaminated by virus. ${ }^{4}$

Reported waterborne outbreaks of virus infection are largely confined to hepatitis type A (infectious hepatitis), 5 mainly because of the dramatic nature of the outbreaks. For example, an explosive outbreak of hepatitis A occurred in New Delhi in December 1955 and January 1956, when after heavy rainfall and flooding sewage contaminated a major water supply for several days. Over 30000 cases of clinical hepatitis among a population of some two million occurred during the next six weeks, despite a presumably high level of immunity in the general population. In the United States 66 waterborne outbreaks of hepatitis A were reported during 1946-71, and another 13 during 1971-4. Many enteric viruses, however, cause inapparent infections, and even clinical illness often shows too variable a picture to enable identification of a common source.

What can we do? Firstly, we now have much evidence that coliforms or enterobacteria are unreliable indicators of viral contamination, and viruses have been found in water in which faecal coliforms are absent. Moreover, treatment methods may destroy faecal bacteria more rapidly than enteric viruses. We therefore need a sensitive virus-indicator system. Suitable methods are indeed being developed-new techniques will detect a single infectious unit in samples as large as 4000 litres of drinking water. Secondly, animal enteric viruses can get into our water supplies, and we need to know more about their effects on human or animal health. Finally, there are important differences in water purification procedures in different parts of the world, and we must establish standard criteria for economic physical and chemical methods of eliminating viruses to maintain the quality of water even when we have to recycle it.

${ }^{1}$ Alvarez, E E, in Viruses in Water, ed G Berg et al. Washington, Public Health Association, 1976.

Melnick, J L, Gerba, C P, and Wallis, C, Bulletin of the World Health ()rganisation, 1978, 56, 499.

"Hoen, R C, et al, fournal of the Environmental Engineering Division of the American Society of Civil Engineers, 1977, 103, 803.

4 Katzenelson, E, Buium, I, and Shuval, H I, Science, 1976, 194, 944.

${ }^{5}$ Zuckerman, A J, Virus Diseases of the Liver. London, Butterworth, 1970.

\section{Preventing immersion hypothermia}

About 25000 people are working in the North Sea in oil and gas production, and perhaps another 5000 or 10000 on merchant and fishing vessels. An unknown number of these people suffer each year from accidental immersion and a few die of hypothermia.

The causes of the incidents are many and complex and such accidents will never be totally preventable. Nevertheless, the hypothermia could be almost eliminated. The most obvious protective measure is adequate clothing. Many so-called "immersion suits" used by sailors, deep-sea fishermen, and particularly passengers in oil-rig helicopters are virtually useless for preventing hypothermia; some are actually dangerous when worn in the water because they are positively buoyant in the legs. A suit that provides excellent protection against atmospheric cold may be quite inadequate for the cold of immersion.

To be really effective in cold water any survival suit must keep the survivor dry. A suit working on the diver's wet-suit principle by trapping a layer of water next to the skin is second best, but not much better than a layer of good, thick woollen clothes. Although a dry suit may allow a little water to seep in from the neck seal, this does not matter provided that the water cannot circulate. 